\title{
Study of the effects of adapted Tango and multidimensional intervention in pREvention of dementia in agiNG: developing healTHy lifestyle programs (STRENGTH Project) — the experimental protocol of a prospective randomised controlled trial
}

\author{
Cinzia Giuli ${ }^{1}$ (1) $\cdot$ Cristina Paoloni ${ }^{1} \cdot$ Elpidio Santillo $^{2} \cdot$ Marta Balietti $^{3} \cdot$ Paolo Fabbietti $^{4} \cdot$ Demetrio Postacchini $^{1}$. \\ Francesco Piacenza ${ }^{5}$
}

Received: 2 September 2019 / Accepted: 5 February 2020 / Published online: 2 March 2020

(c) The Author(s) 2020

\begin{abstract}
Background Dementia represents a key health issue for older adults, with negative consequences on psycho-social and functional status. Treatments that counteract cognitive deficits in mild cognitive impairment (MCI) are needed to prevent or delay it.

Aim To describe the experimental protocol of the STRENGTH Project. This study investigates a multimodal intervention in older adults with MCI to improve cognitive, functional, biochemical and psycho-social aspects.

Methods The prospective randomised controlled trial will enrol 300 subjects with MCI (age $\geq 60$ years). Participants will be randomly assigned to: (a) the experimental group, which will undergo sessions of adapted tango, music therapy, engagement in social activities, cognitive intervention and psycho-education for 6 months or (b) the control group, which will receive psycho-education and advice on healthy lifestyle for 6 months. All outcomes will be analysed before intervention (baseline), immediately after termination (follow-up 1), after 6 months (follow-up 2) and after 2 years (follow-up 3).

Discussion We expect that the findings of this multidisciplinary study will be useful to optimize clinical and psycho-social interventions for improving cognitive and functional status of subjects with MCI.

Conclusions This project could have a meaningful impact on National Health Systems by providing clues on multidisciplinary management of older adults affected by cognitive decline to prevent dementia.
\end{abstract}

Keywords Mild cognitive impairment $\cdot$ Biomarkers $\cdot$ Comprehensive intervention · Adapted tango $\cdot$ Older adults

Cinzia Giuli

c.giuli@inrca.it

1 Geriatrics Operative Unit, Italian National Research Centre on Aging (IRCCS INRCA), Contrada Mossa, 2, 63900 Fermo, Italy

2 Geriatric-Rehabilitative Department, Italian National Research Centre on Aging (IRCCS INRCA), Contrada Mossa, Fermo, Italy

3 Center for Neurobiology of Aging, (IRCCS INRCA), Via Birarelli, Ancona, Italy

4 Unit of Geriatric Pharmacoepidemiology and Biostatistics, Italian National Research Centre on Aging (IRCCS INRCA), Via S. Margherita, Ancona, Italy

5 Advanced Technology Center for Aging Research, Scientific Technological Area, Italian National Research Centre on Aging (IRCCS INRCA), Via Birarelli, Ancona, Italy

\section{Introduction}

Dementia is a chronic and progressive syndrome that affects $5-8 \%$ of general population aged 60 years or more [1]. Dementia damages memory, thinking and behaviour and has negative consequences on psycho-social functioning. Treatments that counteract cognitive deficits in mild cognitive impairment (MCI), a translational state between ageassociated cognitive decline and diagnosed dementia, are needed to prevent or delay it.

Previous studies evidenced that currently available pharmacological therapies are not effective [2], while the use of psycho-social interventions in association with cognitive training and physical activity (PA) has been recognized to improve functional capabilities of older adults, thus positively acting on the onset of neurological diseases [3]. Management of 
cardiovascular and lifestyle-related risk factors is particularly promising [4]. Healthy lifestyle, engagement in regular moderate PA, and involvement in social recreational activities may have protective effects both on mental functions and on risk of dementia [5-7]. Data from our previous "My Mind Project" (Grant no. 154/GR-2009-1584108) showed that subjects affected by MCI had lower level of PA and social participation than age-matched healthy controls and highlighted the potential negative consequences of this situation on psychological conditions and functional status [8]. These findings suggest the necessity to develop multimodal interventions for elderly people with compromised cognition [9]. Indeed, according to the literature $[3,10]$, single-domain trials did not yield long-term effects and, consequently, are not really effective for dementia prevention. Thus, in the STRENGTH Project, we decided to study the effect of a multimodal intervention that associates PA (i.e., adapted tango for non-demented elderly people), cognitive stimulation, music therapy and other psycho-social treatments.

PA programmes based on dance were proved to promote in the elderly population the preservation of functional mobility, the improvement of cognitive processes, mood status and wellbeing and reduction in the fear of falling [11-13]. In particular, a randomised, controlled trial conducted in Italy evidenced specific psycho-social and physical benefits in applying PA based on dance, thanks to the improved balance capability and the reduced risk of falling in the enrolled elders [14]. Nevertheless, more studies regarding dance interventions in the Italian elderly population are needed [11].

Adapted tango was previously tested by other authors, in subjects with different cognitive status, as it was proven to be feasible and safe for improving mobility, physical functions, spatial cognition and memory with maintenance of the positive achievements for 3 months after the end of the intervention $[15,16]$. Moreover, it seems that tango is a particularly innovative approach because of its low PA impact and its effectiveness in promoting positive emotional state and alleviating psychological distress, such as anxiety and depression status in elderly people [17-19]. Depression, anxiety and psychological stress are often observed in MCI subjects due to their consciousness of the cognitive decline; moreover, their subjective memory complaints could intensify the deficit perception worsening their performances [9].

Given this background, the aim of our study is to ameliorate cognition, physical functioning and quality of life in MCI patients, with a potential positive impact on cognitive decline and dementia onset prevention/delay.

\section{Methods}

\section{Study design}

The STRENGTH Project is a prospective randomised controlled trial for the assessment of the effects of a multimodal intervention (i.e., adapted tango, music therapy, engagement in social activities, cognitive stimulation, and psychoeducation for 6 months) in subjects with MCI. The study uses a multidisciplinary approach, has a 3-year duration and includes 3 follow-up phases, as indicated in Fig. 1.

\section{Specific aim 1}

To study the effect of the multimodal intervention on cognitive performances, functional status, and mobility in MCI subjects by objectively measured parameters (baseline vs. follow-up 1, follow-up 2 and follow-up 3). Mobility (i.e., the ability of a subject to move in his/her environment) is often compromised in elderly people as a consequence of falls and other injuries and this could cause disability and reduced social engagement. The identification of approaches that might prevent motor-cognitive impairment would be a pivotal advancement in the correct management of aging people. Mobility will be evaluated by means of balance, walking test and gait speed; motor-cognitive function, defined as a process of coordination of movements finalized to a particular motor goal, will be measured by means of visual-spatial memory task.

The longitudinal monitoring of cognitive decline and dementia onset will also be implemented (follow-up 2 and follow-up 3).

\section{Specific aim 2}

To study the effect of the intervention on psycho-social aspects (e.g., psychological well-being, perceived stress, quality of life, metacognition and mood status), lifestyle characteristics and social network (baseline vs. follow-up 1, follow-up 2 and follow-up 3).

\section{Specific aim 3}

To study the effect of the intervention on specific biomarkers (baseline vs. follow-up 1, follow-up 2 and follow-up 3).

\section{Experimental design aim 1}

Neuropsychological as well as cognitive and clinical assessment, including cardiovascular, anthropometric, and functional evaluation (e.g., blood pressure, body mass index, 
Flow Chart of STRENGTH Project
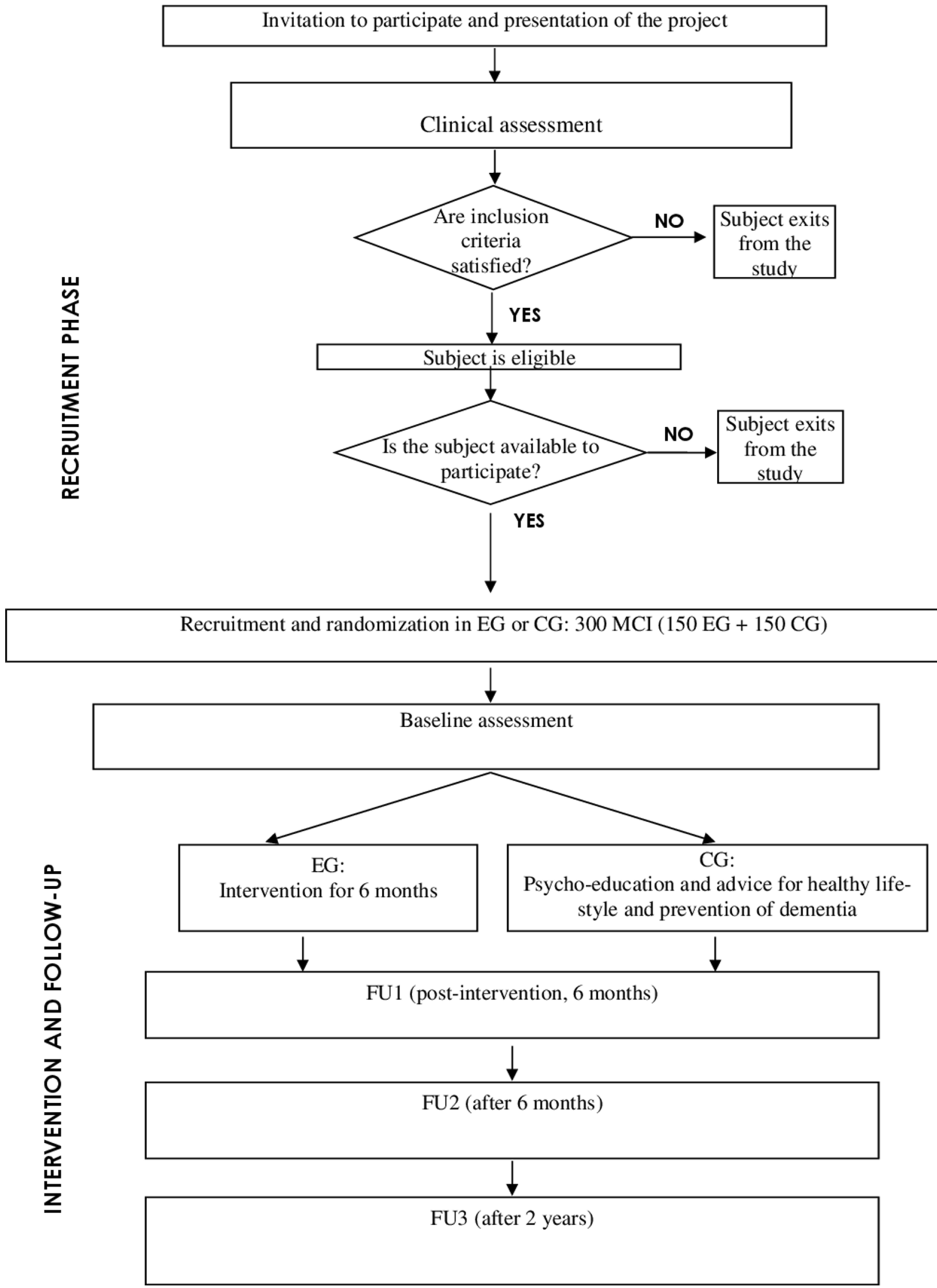

Fig. 1 Flowchart of strength project 
ultrasound heart examination through standard echocardiogram, 6-min walk test, gait speed, Berg Balance Scale, handgrip strength, Physical Activity Scale for the Elderly, basic activities of daily living and instrumental activities of daily living) will be carried out (Table 1).

\section{Experimental design aim 2}

Enrolled subjects will undergo a detailed protocol of tests and questionnaires to evaluate the following aspects: psychological well-being, perceived stress, quality of life, metacognition, mood status, lifestyle characteristics and social network (Table 1) [20-39].

\section{Experimental design aim 3}

The following parameters will be evaluated (details are reported in Fig. 2):

(a) BDNF and ceruloplasmin protein amounts in plasma and their mRNA expression in peripheral blood monuclear cells (PBMC), as the role of peripheral leukocyte

Table 1 Clinical, functional, and neuropsychological assessment

\begin{tabular}{l}
\hline Instruments \\
\hline Cognitive assessment \\
Clinical Dementia Rating Scale_CDR [20] \\
Memory Complaint Questionnaire-MAC-Q [21] \\
Montreal cognitive assessment-MoCA [22] \\
Mini mental state examination-MMSE [23] \\
Rey auditory verbal learning test-RAVLT [24] \\
Phonological verbal fluency-PVF [24] \\
Supra-span of Corsi [25] \\
Semantic verbal fluency-SVF [26] \\
Attentive matrices [26] \\
Trail making test A-B-TMT A-B [27] \\
Psychological assessment \\
Depression Anxiety Stress Scale-DASS [28, 29] \\
Geriatric Depression Scale-15-GDS-15 [30] \\
Psychological Well-Being Scales—PWB [31, 32] \\
SF-36 (36 health survey) [33] \\
Functional assessment \\
Basic activities of daily living—ADL [34] \\
Instrumental activities of daily living—IADL [35] \\
Berg Balance Scale-BBS [36] \\
6-min walking test-6MWT [37] \\
Social network \\
Lubben Social Network Scale—LSNS [38] \\
Lifestyle characteristics and physical activity \\
Physical Activity Scale for the Elderly—PASE [39] \\
Lifestyle Questionnaire \\
\hline
\end{tabular}

dysfunctions in Alzheimer's disease (AD) development $[40,41]$ is well known;

(b) mRNA expression in PBMC of 84 key genes involved in $\mathrm{AD}$ physiopathology (i.e., beta-amyloid generation, oligomerization, clearance, and degradation; cytoskeleton regulators; synaptic formation; lipid metabolism; apoptosis; cell cycle; cell signalling molecules; transcriptional regulation; oxidative stress; proteases and protease inhibitors);

(c) plasma copper, zinc, and other important micronutrients;

(d) a panel of the most common laboratory parameters.

\section{Recruitment and screening phase}

The project started on 1 August 2019 and will last until 31 July 2022. The protocol was approved by the local ethics committee (Bioethics Advisory Committee of IRCCS INRCA, Ancona, Italy; code no. 18006). Three hundred community-dwelling older adults (age $\geq 60$ years) with diagnosis of MCI will be enrolled in the Evaluation of dementia's disease Unit of IRCCS INRCA Hospital in Fermo. MCI will be diagnosed by an extended neuropsychological, clinical and functional evaluation, as well as by neuroimaging and laboratory tests according to diagnostic guidelines [42]. The subjects' screening will be performed during routine clinical visits applying specific inclusion/exclusion criteria as explicated below. Written informed consent will be obtained from all subjects.

\section{Randomisation}

The selected subjects will be randomly allocated to the experimental group (EG, $n=150$ ) or to the control group (CG, $n=150)$ using a randomisation list.

\section{Inclusion and exclusion criteria}

The inclusion criteria comprise: (a) diagnosis of MCI according to the guidelines of the National Institute of Aging [42]; (b) age 60 years or older, (c) availability during the intervention and testing phases, (d) presence of a caregiver; (e) capability to sign the informed consent.

Exclusion criteria include: (a) presence of sensory-physical deficits that would prevent participation in the study; (b) diagnosis of dementia; (c) presence of serious medical and/ or psychiatric conditions.

\section{Sample size calculation}

It was based on the null hypothesis that proportion of "success" and "failure" of the intervention is equal in the two groups (i.e., $\mathrm{EG}$ and $\mathrm{CG}$ ), such as $\mathrm{OR}=1$. 
Analysis in Whole blood

Haemochrome

- RBC

- WBC

- Platelets

- Haematocrit

- Haemoglobin

- MCV

Coagulation

- PT

- PTT

Analysis in Peripheral blood mononuclear cells (PBMC)

- mRNA expression of 84 genes involved in AD development

Pathways analysed

- Beta-Amyloid Generation, Oligomerization, Clearance \& Degradation

- Cytoskeleton Regulators

- Synaptic Formation

- Lipid Metabolism

- Apoptosis

- Cell Cycle

- Cell Signalling Molecules

- Transcriptional Regulation

- Oxidative Stress and Proteases \& Protease Inhibitors

\section{Analysis in plasma}

Biomolecules

- Glucose

- Ceruloplasmin

- BDNF

\section{Liver function}

- ALT, AST

- Albumin

- Bilirubin

- Total proteins

Kidney function

- BUN

- Creatinine

- e-GFR (CKD-EPI)

Lipid pathway

- Total Cholesterol

- HDL

- LDL

- Triglycerides

Trace elements and Electrolytes

- $\mathrm{Cu}, \mathrm{Zn}, \mathrm{Se}, \mathrm{Fe}$

- $\mathrm{K}, \mathrm{Ca}, \mathrm{Na}, \mathrm{Mg}$
Fig. 2 The panel represents all the biomarkers that will be analysed in the STRENGTH Project for each tissue (i.e., whole blood, plasma, and peripheral blood mononuclear cells). $R B C$ red blood cells, $W B C$ white blood cells, $M C V$ mean corpuscular volume, $P T$ prothrombin time, $P T T$ partial thromboplastin time, $B D N F$ brain-derived neurotrophic factor, $A L T$ alanine amino transferase, $A S T$ aspartate amino transferase, $B U N$ blood urea nitrogen, $e$-GFR estimated glomerular filtration rate, $C K D$-EPI chronic kidney disease epidemiology collaboration, $H D L$ high-density lipoprotein, $L D L$ low-density lipoprotein, $\mathrm{Cu}$ copper, $\mathrm{Zn}$ Zinc, Se selenium, $\mathrm{Fe}$ iron, $\mathrm{Ca}$ calcium, $\mathrm{Mg}$ magnesium, $N a$ sodium, $K$ potassium, $A D$ Alzheimer's Disease
Success and failure were defined on the basis of significant variation in the primary outcomes. Considering an $\mathrm{OR}=1.5$ indicative of an effect of the intervention, a total sample of 300 subjects allows us to reach a $90 \%$ of statistical power using logistic regression at the 5\% level (two tailed). Drop-out rate was set at $20 \%$.

\section{Intervention}

A group approach will be used. The intervention will be focused not only on cognitive enhancement, but also on several other aspects, such as advice and psycho-education about healthy lifestyle strategies as well as engagement in leisure activities. EG will be followed by a multidisciplinary team (i.e., psychologists, physicians, nurses, tango instructors and music therapist).

Multimodal intervention will consist in a 20-class syllabus of 180-min session, once a week, including $90 \mathrm{~min}$ of adapted tango in accordance to guidelines for physical activity in aging and literature [15], and, in the same session, further 90 min of comprehensive cognitive intervention for 6 months. The intervention will maximize social participation. Tango will be adapted for older adults with mild cognitive decline and possible mild balance and musculoskeletal impairment. Tango classes will be carried out in a ballroom dancing, led by two experienced and qualified Argentine instructors, with years of certified proficiency and experience in teaching adapted tango methods to elderly people. Classes will take into account the engagement of participants 
and feasibility. Each class will include about 25 subjects who will dance in pairs with other participants, including instructors, relatives and caregivers. Classes will begin with a phase of warm-up consisting of postural alignment and breathing. The main phase of dance will include enhancement exercises and addition of novel simple steps at each class with varied speeds and rhythms, individually and in pairs, focused on body awareness to educate subjects on prevention of falls, improving mobility, foot placement, whole body coordination, postural control, balance, self-confidence and attention to dance partner. In the last phase, participants can share their experience or ask questions. Possible adverse events will be tracked.

Cognitive intervention will include restorative and compensatory cognitive stimulation, learning mnemonics strategies and music therapy, and will be carried out by two trained psychologists and a music therapist. Intervention will use a metacognitive and a motivational approach applied to subjects with MCI, as described in previous studies [43, 44]. Participants will be also required to perform home exercises, which will include some cognitive stimulation exercises, assigned at the end of each class. Homework will be reviewed by the psychologist during the next class.

An example syllabus of classes is reported in Table 2.

Possible adverse events, adherence to the study and participants' attrition will be monitored by the principal investigator, adapted tango teachers and other staff members involved in the intervention.

To avoid bias effect of the intervention, GC will receive psycho-education and advice for healthy lifestyle as well as suggestions about the prevention of dementia once a month for 6 months. This group will receive the same clinical monitoring of the general health status, neuropsychological assessment, and cardiovascular parameters of EG. Moreover, they will be informed about the results and possible benefits of intervention, so they could have the possibility to start classes of tango therapy at the end of the study, if they so wish. Indeed, at the end of the study, we expect that this type of program will become a new service offered by IRCCS INRCA to elderly persons with an increased risk of cognitive decline.

\section{Primary outcome: success of the intervention}

The response to the intervention will be evaluated using performance in the Corsi test [25]: an increase of at least 1 point will indicate a success based on the enhancement of the spatial learning processes.

\section{Secondary outcomes}

They include:

- the assessment of the intervention effects on lifestyle characteristics as well as psychological, functional and health status;

- the assessment of the intervention effects on mobility;

- the investigation of the conversion rate from MCI to dementia;

- the identification of biomarkers able to mirror the intervention effects.

\section{Statistical analysis}

All data will be collected in a database. A descriptive analysis will be performed to assess the distribution of variables and the presence of possible outliers. The latter will be checked and identified separately and then excluded from the analysis. For normally distributed variables, the comparisons between EG and CG at baseline will be carried

Table 2 Example syllabus of classes

Classes

First class Welcome to participants. Subjects can share their experience about cognitive impairment or ask questions.

Second class Welcome to participants. Questions of participants.

Third class

Welcome to participants. Questions of participants.

Revision of homework assigned the previous week.

Fourth class Welcome to participants. Questions of participants. Revision of homework assigned the previous week.
75 min of psycho-education about healthy 90 min of dance. Explanation about the lifestyle aimed to prevention of dementia aim of adapted tango and other pathologies

75 min of psycho-education and health advice about the cognitive enhancement for prevention and management of cognitive disorders. Assignment of homework

75 min of cognitive stimulation aimed to learning of mnemonic strategies.

Assignment of homework

75 min of music therapy. Assignment of homework
90 min of adapted tango, as indicated in "Methodology"

90 min of adapted tango, as indicated in "Methodology"

90 min of adapted tango, as indicated in "Methodology" 
out by $t$ Student test or Chi-square test, while those between baseline and follow-up phases by paired sample $t$ test. For non-normally distributed variables, non-parametric tests will be used. Correlation analysis will be performed to evaluate the relationships among variables and general linear models and repeated measures ANOVA will be used to assess the intervention effects adjusted for confounding variables (e.g., gender, age classes, marital status, schooling, drugs, and other variables that will emerge after analysis of secondary outcomes). The SPSS software will be employed.

\section{Discussion and conclusion}

The main expected result is the development of a clinical and psycho-social intervention that is able to reduce age-related disability (both physical and cognitive), improve quality of life, well-being and socialization, and counteract cognitive decline and dementia development. The treatment will include different domains, such as rehabilitation and enhancing therapy, monitoring of health status and cardiovascular parameters, psycho-education and neuropsychological stimulation. Socio-relational aspects with a positive impact on psychological status will also be taken into account.

The innovative topic is the use of adapted tango in our multimodal intervention: it seems to enhance physicalcognitive aspects because of the required alternation of attention among postural control, current balance, cognition-challenging step, and spatial memory. In particular, previous studies have evidenced that this kind of dance is sustainable and acceptable by independent elderly people, because it is a feasible PA [13]. Benefits were observed in activities of daily living (ADL) performance and quality of life of older adults [15] and, its benefits known, it was successfully applied to patients with different neurological conditions $[16,45]$. A recent paper evidenced that tango has many favourable therapeutic characteristics, but it is important to check and evaluate the progress and the adverse events during the intervention to prevent side effects as well as to optimize tolerance and adherence [18]. Few studies described the occurrence of adverse events or untoward results, so they will be monitored. Classes will be well-structured, to promote socialization, feasibility, and engagement. Therefore, according to the patient's status, this therapeutic intervention should be built by a multidisciplinary team, which is going to include clinical staff, in accordance to medical concept [18]. In our study, an important achievement will be the creation of a multidisciplinary, highly specialized team of professionals who will work together to improve the quality of life of the older adults affected by MCI. These subjects at high risk of developing dementia could be treated early with a focused intervention, to slow the rate of conversion to dementia or prevent the conversion. Moreover, the evaluation of functional aspects, not only in terms of preservation of functional independence in daily life activities, but also in terms of reduction of falls, enhanced capacity to walk/move, maintain gait speed and balance without or with minimal assistance, and improvement of cognitive and psycho-social functions, will be used to identify the benefits of intervention at the end of the 3 years.

We will deepen the consequences on community-dwelling older subjects with $\mathrm{MCI}$ and will test a longer protocol (i.e., 6 months) not previously considered. Another key element is the evaluation of engagement in psycho-social and relational activities related not only to dance but also to cognitive stimulation, music therapy included.

The multidisciplinary approach will be strengthened by the evaluation of several biomarkers: this could help us better define the intervention efficacy. Indeed, in a previous study (i.e., My Mind Project), this approach allowed operators to display the positive effect of a comprehensive intervention based on cognitive stimulation on specific biological dysfunctions due to $\mathrm{MCI}$ or $\mathrm{AD}$, such as platelet total phospholipases A2 activity [46], lymphocytic brain-derived neurotrophic factor (BDNF) mRNA expression [47], as well as plasma BDNF concentration [48].

This project might have a significant impact on National Health Systems by providing a multidisciplinary management of MCI subjects to slow down cognitive decline and potentially reduce the conversion rate to dementia. In Italy and worldwide, dementia represents a pivotal public health issue that negatively affects older persons' independence, thus creating medical, social, and economic burdens. It is essential to define specific interventions to increase quality of life and well-being in the elderly: such type of programs could become a new service offered by the health services to older adults.

Funding This study was supported by "Ricerca Finalizzata" funding from the Italian Ministry of Health Grant and the Marche Region (grant number GR-2016-02363041) to Cinzia Giuli and IRCCS INRCA.

\section{Compliance with ethical standards}

Conflict of interest On behalf of all authors, the corresponding author states that there is no conflict of interest. All authors declare that they have no conflict of interest.

Statement of human and animal rights All procedures performed in studies involving human participants were and will be in accordance with the ethical standards of the institutional and/or national research committee (IRCCS INRCA, Bioethics Advisory Committee, Ancona, Italy, approval code 18006) and with the 1964 Helsinki declaration and its later amendments or comparable ethical standards. The final protocol has been submitted to the local ethics committee. 
This article does not contain any studies with animal performed by any of the authors.

Informed consent Informed consent will be obtained from all participants included in the study.

Open Access This article is licensed under a Creative Commons Attribution 4.0 International License, which permits use, sharing, adaptation, distribution and reproduction in any medium or format, as long as you give appropriate credit to the original author(s) and the source, provide a link to the Creative Commons licence, and indicate if changes were made. The images or other third party material in this article are included in the article's Creative Commons licence, unless indicated otherwise in a credit line to the material. If material is not included in the article's Creative Commons licence and your intended use is not permitted by statutory regulation or exceeds the permitted use, you will need to obtain permission directly from the copyright holder. To view a copy of this licence, visit http://creativecommons.org/licenses/by/4.0/.

\section{References}

1. World Health Organization. Global action plan on the public health response to dementia 2017-2025. https://www.who.int/ mental_health/neurology/dementia/action_plan_2017_2025/en/. Accessed 2017

2. Rodakowski J, Saghafi E, Butters MA et al (2015) Non-pharmacological interventions for adults with mild cognitive impairment and early stage dementia: an updated scoping review. Mol Aspects Med 43-44:38-53

3. Ngandu T, Lehtisalo J, Solomon A et al (2015) A 2 year multidomain intervention of diet, exercise, cognitive training, and vascular risk monitoring versus control to prevent cognitive decline in at-risk elderly people (FINGER): a randomised controlled trial. Lancet 385:2255-2263

4. Orgeta V, Mukadam N, Sommerlad A et al (2019) The lancet commission on dementia prevention, intervention, and care: a call for action. Ir J Psychol Med 36:85-88

5. Galenkamp H, Gagliardi C, Principi A et al (2016) Predictors of social leisure activities in older Europeans with and without multimorbidity. Eur J Ageing 13:129-143

6. Andrews RM, Tan EJ, Varma VR et al (2017) Positive aging expectations are associated with physical activity among urbandwelling older adults. Gerontologist 57:S178-S186

7. Petersen RC, Lopez O, Armstrong MJ et al (2018) Practice guideline update summary: mild cognitive impairment: report of the guideline development, dissemination, and implementation subcommittee of the American Academy of Neurology. Neurology 90:126-135

8. Gagliardi C, Papa R, Postacchini D et al (2016) Association between cognitive status and physical activity: study profile on baseline survey of the My Mind Project. Int J Environ Res Public Health 13:585

9. Giuli C, Papa R, Lattanzio F et al (2016) The effects of cognitive training for elderly: results from My Mind Project. Rejuvenation Res 19:485-494

10. Rosenberg A, Ngandu T, Rusanen M et al (2018) Multidomain lifestyle intervention benefits a large elderly population at risk for cognitive decline and dementia regardless of baseline characteristics: the FINGER trial. Alzheimers Dement 14:263-270

11. Veronese N, Maggi S, Schofield P et al (2017) Dance movement therapy and falls prevention. Maturitas 102:1-5
12. McKinley P, Jacobson A, Leroux A et al (2008) Effect of a community-based Argentine Tango dance program on functional balance and confidence in older adults. J Aging Phys Act 16:435-453

13. Brustio PR, Liubicich ME, Chiabrero M et al (2018) Dancing in the golden age: a study on physical function, quality of life, and social engagement. Geriatr Nurs 39:635-639

14. Federici A, Bellagamba S, Rocchi MB (2005) Does dance-based training improve balance in adult and young old subjects? A pilot randomized controlled trial. Aging Clin Exp Res 17:385-389

15. Hackney ME, Byers C, Butler G et al (2015) Adapted Tango improves mobility, motor-cognitive function, and gait but not cognition in older adults in independent living. J Am Geriatr Soc 63:2105-2113

16. Zafar M, Bozzorg A, Hackney ME (2017) Adapted Tango improves aspects of participation in older adults versus individuals with Parkinson's disease. Disabil Rehabil 39:2294-2301

17. Pinniger R, Brown RF, Thorsteinsson EB et al (2012) Argentine Tango dance compared to mindfulness meditation and a waiting-list control: a randomised trial for treating depression. Complement Ther Med 20:377-384

18. Youngsoon K, IC SK, Geunwoong N (2018) Tango therapy: current status and the next perspective. J Clin Rev Case Rep $3: 1-5$

19. Koch SC, Riege RFF, Tisborn K, Biondo J et al (2019) Effects of dance movement therapy and dance on health-related psychological outcomes. A meta-analysis update Front Psychol 10:1806

20. Hughes CP, Berg L, Danziger WL et al (1982) A new clinical scale for the staging of dementia. Br J Psychiatry 140:566-572

21. Crook TH, Feher EP, Larrabee GJ (1992) Assessment of memory complaint in age-associated memory impairment: the MAC-Q. Int Psychogeriatr 4:165-176

22. Nasreddine ZS, Phillips NA, Bédirian V et al (2005) The Montreal Cognitive Assessment, MoCA: a brief screening tool for mild cognitive impairment. J Am Geriatr Soc 53:695-699

23. Folstein MF, Folstein SE, McHugh PR (1975) "Mini-mental state": a practical method for grading the cognitive state of patients for the clinician. J Psychiatr Res 12:189-198

24. Carlesimo GA, Caltagirone C, Gainotti G et al (1996) The mental deterioration battery: normative data, diagnostic reliability and qualitative analyses of cognitive impairment. Eur Neurol 36:378-384

25. Orsini A, Grossi D, Capitani E et al (1987) Verbal and spatial immediate memory span: normative data from 1355 adults and 1112 children. Ital J Neurol Sci 8:537-548

26. Spinnler H, Tognoni G (1987) Standardizzazione e taratura italiana di test neuropsicologici. Ital J Neurol Sci 6:35-38

27. Amodio P, Wenin H, Del Piccolo F et al (2002) Variability of trail making test, symbol digit test and line trait test in normal people. A normative study taking into account age-dependent decline and sociobiological variables. Aging Clin Exp Res 14:117-131

28. Lovibond PF, Lovibond SH (1995) The structure of negative emotional states: comparison of the Depression Anxiety Stress Scales (DASS) with the Beck Depression and Anxiety Inventories. Behav Res Ther 33:335-343

29. Bottesi G, Ghisi M, Altoè $G$ et al (2015) The Italian version of the Depression Anxiety Stress Scales-21: factor structure and psychometric properties on community and clinical samples. Compr Psychiatry 60:170-181

30. Sheikh JI, Yesavage JA (1986) Geriatric Depression Scale (GDS): recent evidence and development of a shorter version. Clinical Gerontology: a guide to assessment and intervention. The Haworth Press Ltd, New York, pp 165-173

31. Ryff CD, Singer B (1996) Psychological well-being: meaning, measurement, and implications for psychotherapy research. Psychother Psychosom 65:14-23 
32. Ruini C, Ottolini F, Rafanelli C et al (2003) La validazione italiana delle Psychological Well-being Scales (PWB). Riv Psichiatr 38:117-130

33. Apolone G, Mosconi P, Ware JE (1997) Questionario sullo stato di salute SF-36. Guerini e Associati, Verona

34. Katz S, Ford AB, Moskowitz RW et al (1963) Studies of illness in the aged. The index of ADL: a standardized measure of biological and psychosocial function. JAMA 185:914-919

35. Lawton MP, Brody EM (1969) Assessment of older people: selfmaintaining instrumental activities of daily living. Gerontologist 9:179-186

36. Berg K, Wood-Dauphinee SL, Williams JL (1992) Measuring balance in the elderly: validation of an instrument. Can J Public Health 83:S7-S11

37. Guyatt GH, Sullivan MJ, Thompson PJ et al (1985) The 6-minute walk: a new measure of exercise capacity in patients with chronic heart failure. Can Med Assoc J 132:919-923

38. Lubben J, Blozik E, Gillmann G et al (2006) Performance of an abbreviated version of the Lubben Social Network Scale among three European community-dwelling older adult populations. Gerontologist 46:503-513

39. Washburn RA, McAuley E, Katula J et al (1999) The Physical Activity Scale for the Elderly (PASE): evidence for validity. J Clin Epidemiol 52:643-651

40. Naert G, Rivest S (2013) A deficiency in CCR2+ monocytes: the hidden side of Alzheimer's disease. J Mol Cell Biol 5:284-293

41. Vérité J, Page G, Paccalin M et al (2018) Differential chemokine expression under the control of peripheral blood mononuclear cells issued from Alzheimer's patients in a human blood brain barrier model. PLoS ONE 13:e201232
42. Albert MS, DeKosky ST, Dickson D et al (2011) The diagnosis of mild cognitive impairment due to Alzheimer's disease: recommendations from the National Institute on Aging and Alzheimer's Association Workgroup. Alzheimers Dement 7:270-279

43. Giuli C, Fabbietti P, Paoloni C et al (2016) Subjective memory complaints in Italian elderly with mild cognitive impairment: implication of psychological status. Neurol Sci 37:1153-1157

44. Zahodne LB, Watson CW, Seehra S et al (2018) Positive psychosocial factors and cognition in ethnically diverse older adults. $\mathrm{J}$ Int Neuropsychol Soc 24:294-304

45. Patterson KK, Wong JS, Prout EC et al (2018) Dance for the rehabilitation of balance and gait in adults with neurological conditions other than Parkinson's disease: a systematic review. Heliyon 4:e00584

46. Balietti M, Giuli C, Fattoretti P et al (2016) Cognitive stimulation modulates platelet total phospholipases A2 activity in subjects with mild cognitive impairment. J Alzheimers Dis 50:957-962

47. Casoli T, Giuli C, Balietti M et al (2014) Effect of cognitive training on the expression of brain-derived neurotrophic factor in lymphocytes of mild cognitive impairment patients. Rejuvenation Res 17:235-238

48. Balietti M, Giuli C, Fattoretti P et al (2017) Effect of a comprehensive intervention on plasma BDNF in patients with Alzheimer's Disease. J Alzheimers Dis 57:37-43

Publisher's Note Springer Nature remains neutral with regard to jurisdictional claims in published maps and institutional affiliations. 